\title{
Pseudo-Banach algebras
}

by

G. R. ALLAN (Leeds, England), H. G. DALES (Glasgow, Scotland) J. P. MCCLURE (Ontario, Canada)

Abstract. A pseudo-Banach algebra is, algebraically, an inductive limit of Banach algebras. In this paper it is shown that a great deal of the general theory of commutative Banach algebras extends to commutative pseudo-Banach algebras and also that this latter class includes many interesting examples not contained in the class of Banach algebras.

Introduction. The definition of a pseudo-Banach algebra arises from work of the first named author on spectral theory for locally convex algebras [1], and in particular from the notion of a bound structure in an algebra. The importance in this context of a structure analogous to a system of bounded sets has also been recognized by Waelbroeck, who has developed a theory of 'algèbres à bornés complètes' in a rather different direction. (See for example [12].)

The pseudo-Banach algebras are characterized immediately from their definition as the (algebraic) inductive limits of inductive systems of Banach algebras and continuous monomorphisms. Their interest lies in two facts: the extent to which their properties parallel those of Banach algebras, and the fact that the pseudo-Banach algebras form a considerably larger class than the Banach algebras. We shall see that a commutative pseudo-Banach algebra with identity has a space of characters which is non-empty and compact in the weak*-topology, that every maximal ideal is the kernel of a character, and that the analytic functional calculus holds for such algebras in a form analogous to the strong functional calculus for Banach algebras. Moreover, the pseudo-Banach algebras are characterized, among commutative algebras with identity and compact space of characters, by the existence of a functional calculus. Thus, pseudo-Banach algebras form the natural setting for such applications of the functional calculus as Rossi's local peak set theorem and the Arens-Royden theorem, and we shall see that such results hold for pseudo-Banach algebras with only minor modifications to the Banach algebra proofs. 
In this paper, we shall consider only linear associative algebras over the complex field $C$. Also, although the definition clearly applies in general, we shall throughout restrict ourselves to commutative algebras. If an identity were not present, we could adjoin one as in [1], Proposition (2.8), and so we consider only algebras with identity.

The second named author acknowledges the financial support of a Science Research Council studentship, and the third named author that of a Commonwealth Scholarship. The paper was written while the three authors were at the University of Cambridge.

1. Definitions and basic properties.

Definition 1.1. Let $A$ be a commutative algebra with identity $e$. $A$ bound structure for $A$ is a non-empty collection $\mathscr{B}$ of subsets of $A$ such that

(i) $\mathrm{B}$ is absolutely convex, $B^{2} \subset B, e \in B$, for each $B$ in $\mathscr{B}$,

(ii) given $B_{1}, B_{2}$ in $\mathscr{B}$, there exists $B_{3}$ in $\mathscr{B}$ and $\lambda>0$ such that $B_{1} \cup B_{2} \subset \lambda B_{3}$.

$(A, \mathscr{B})$ is then a bound algebra.

For $B$ in $\mathscr{B}$, let $A(B)=\{\lambda b: \lambda \in C, b \in B\}$; in view of (i), $A(B)$ is the subalgebra of $A$ generated by $B$. The Minkowski functional of $B$ defines a submultiplicative semi-norm $\|\cdot\|_{B}$ on $A(B)$. If each $\|\cdot\|_{B}$ is a norm, and if $A(B)$ is a Banach algebra with respect to $\|\cdot\|_{B}$, then $(A ; \mathscr{B})$ is complete.

From (ii), $A_{0}=\bigcup\{A(B): B \epsilon \mathscr{B}\}$ is a subalgebra of $A$. If $A$ is complete, and if $A=A_{0}$, then $A$ is a pseudo-Banach algebra.

Proposition 1.2. An algebra $A$ is pseudo-Banach with respect to some bound structure if and only if $A$ is isomorphic with the inductive limit of an inductive system $\left\{A_{a} ; \pi_{\beta \alpha}: \alpha, \beta \in \Lambda, \alpha \leqslant \beta\right\}$ of Banach algebras with identity and continuous unital monomorphisms.

Proof. Let $A$ be a pseudo-Banach algebra and let the bound structure be indexed by a set $\Lambda$. The set $\Lambda$ is directed upwards by the relation $\leqslant$ defined by ' $\alpha \leqslant \beta$ if and only if $B_{\alpha} \subset \lambda B_{\beta}$ for some $\lambda>0$ '. Write $A_{\alpha}$ for $A\left(B_{a}\right)$ and $\|\cdot\|_{a}$ for the norm on $A_{\alpha}$. For $\alpha \leqslant \beta, A_{\alpha} \subset A_{\beta}$ and the inclusion map $\pi_{\beta \alpha}$ is a continuous unital monomorphism. It is clear that $\left\{A_{a}\right.$; $\left.\pi_{\beta \alpha}\right\}$ is the required inductive system.

Conversely, if $\left\{A_{\alpha} ; \pi_{\beta a}\right\}$ is such an inductive system, the unit balls of the algebras $A_{a}$ (when identified with subalgebras of $A$ ) can be taken for the members of a bound structure with respect to which $A$ is pseudoBanach.

The notation for the bound structure used in the above proposition will be used for the remainder of the paper.

A character on a pseudo-Banach algebra $A$ is a homomorphism of $A$ onto $C$. Let $\left(X_{A}, \sigma\left(X_{A}, A\right)\right)$ be the space of characters on $A$ with the relative weak-* topology, and for $\alpha$ in $A$, let $\left(X_{\alpha}, \sigma\left(X_{a}, A_{a}\right)\right)$ be the carrier space of the Banach algebra $A_{\alpha}$. Define $\varrho_{\alpha \beta}: X_{\beta} \rightarrow X_{\alpha}(\alpha \leqslant \beta)$ by restriction. It is clear that $\left\{X_{\alpha} ; \varrho_{\alpha \beta}: \alpha, \beta \in \Lambda, \alpha \leqslant \beta\right\}$ is a projective limit system of compact Hausdorff spaces and continuous mappings.

Lencma 1.3. $X_{A}$ is homeomorphic to limproj $\left\{X_{a} ; \varrho_{\alpha \beta}\right\}$.

Proof. For each $\alpha$, let $\varrho_{\alpha}: X_{A} \rightarrow X_{\alpha}$ be defined by restriction, and let $\varrho(x)=\left(\varrho_{\alpha}(x)\right) \epsilon \Pi X_{\alpha}$ for $x$ in $X_{A}$. Then $\varrho$ is a continuous bijection onto the projective limit $\mathrm{L}$. We show that $\varrho^{-1}$ is continuous. For $x \in X_{A}$, a basic neighbourhood of $x$ is

$$
U=\left\{y \in X_{A}:\left|y\left(a_{i}\right)-x\left(a_{i}\right)\right|<1, i=1, \ldots, n\right\},
$$

where $a_{1}, \ldots, a_{n} \in A$. Choose $\beta$ such that $a_{1}, \ldots, a_{n} \in A_{\beta}$. If

$$
V=\left\{\left(x_{\alpha}\right) \epsilon L:\left|x_{\beta}\left(a_{i}\right)-\varrho_{\beta}(x)\left(a_{i}\right)\right|<1, i=1, \ldots, n\right\},
$$

then $V$ is a neighbourhood of $\varrho(x)$ in $L$ and $V \subset \varrho(U)$.

Thus, $\varrho$ is a homeomorphism and the lemma is proved.

CoRollakY 1.4. $X_{A}$ is a non-empty compact Hausdorff space.

Proof. See [1], Proposition 6.2.

Proposition 1.5. Let $J$ be any ideal in the pseudo-Banach algebra $\boldsymbol{A}$. Then $J$ is a proper ideal if and only if $J \subset \operatorname{ker} x$ for some $x$ in $X_{\mathcal{A}}$.

Proof. The sufficiency is clear.

For the necessity, suppose that $J$ is a proper ideal in $A$. For each $\alpha \in A$, let $J_{\alpha}=J \cap A_{\alpha}$ and let $K_{\alpha}=\left\{x_{\alpha} \in X_{\alpha}: x_{\alpha} \mid J_{\alpha}=0\right\}$. Then $K_{\alpha}$ is a compact subset of $X_{a}$ which is non-empty because $J_{a}$ is a proper ideal of $A_{\alpha}-$ it cannot contain the identity. Also, $\varrho_{\alpha \beta}\left(K_{\beta}\right) \subset K_{\alpha}$ for $\alpha \leqslant \beta$. Thus, $K=\operatorname{limproj}\left\{K_{\alpha} ; \varrho_{\alpha \beta}\right\}$ is non-empty, and $J \subset \operatorname{ker} x$ for any $x \in K$. This completes the proof of the proposition.

Corollary 1.6. If $J$ is a maximal ideal in $A$, then $J=\operatorname{ker} x$, some $x \in X_{A}$.

Corollary 1.7 (Gelfand-Mazur Theorem). If $A$ is a field, then $\boldsymbol{A}$ is isomorphic to $\boldsymbol{C}$.

With each $a$ in the pseudo-Banach algebra $A$, associate a complexvalued function $\hat{a}$ on $\dot{X}_{4}$ by

$$
\hat{a}(x)=x(a) \quad\left(x \in X_{A}\right) .
$$

Each such function is continuous on $X_{A}$. The map $a \rightarrow \hat{a}$ is a homomorphism, the Gelfand representation of $A$. In view of 1.6 the (Jacobson) radical of $A$ is equal to the ideal $R=\left\{a \in A: x(a)=0, x \in X_{A}\right\}$. The algebra is semi-simple if $R=\{0\}$. In this case, the Gelfand representation is a monomorphism, and $A$ can be identified with an algebra of continuous functions on $X_{A}$. 
Let $X$ be a compact Hausdorff space and let $C(X)$ be the algebra of continuous complex-valued functions on $X$. A function algebra is a subalgebra of $C(X)$ which separates the points of $X$ and which contains the constants. The algebra is natural if every character is given by evaluation at a point of $X$. We shall consider (natural) pseudo-Banach function algebras in Theorem 3.8 .

Defintrion 1.8. Let $A_{1}, A_{2}$ be pseudo-Banach algebras with bound structures $\mathscr{B}_{1}, \mathscr{B}_{2}$, respectively. $A$ unital homomorphism $\mu: A_{1} \rightarrow A_{2}$ is called bound-preserving if, for every $B \in \mathscr{B}_{1}$, there exist $B_{\mu} \in \mathscr{B}_{2}$ and $r>0$ such that $\mu(B) \subset r B_{\mu}$.

The bound-preserving homomorphisms are the 'structurepreserving' homomorphisms for pseudo-Banach algebras. It is clear that the composition of bound-preserving homomorphisms is bound-preserving.

\section{Examples.}

EXAMPLE 2.1. BANACH ALGEBRAS. Of course, every commutative Banach algebra with identity is a pseudo-Banach algebra with respect to the bound structure consisting of the unit ball of the algebra.

EXAMPLE 2.2. LOCALLY CONVEX ALGEBRAs. A locally convex algebra is an associative linear algebra $A$ equipped with a topology $\tau$ such that $(A, \tau)$ is a Hausdorff locally convex space and such that multiplication in the algebra is separately continuous.

Denote by $\mathscr{B}$ the collection of all subsets $B$ of $A$ such that

(i) $B$ is absolutely convex, $B^{2} \subset B, e \in B$,

(ii) $B$ is closed and bounded.

If $(A, \tau)$ is complete (or sequentially complete or quasi-complete), then $(A ; \mathscr{B})$ is a complete bound algebra.

The algebra $(A ; \mathscr{B})$ is pseudo-Banach if and only if every element is bounded, i. e., for each element $a$ of $A$, there is a non-zero complex number $\lambda$ for which the set $\left\{(\lambda a)^{n}: n=1,2, \ldots\right\}$ is a bounded subset of $A$.

For details of the above remarks, see [1].

EXAMPLE 2.3. LOCALIY MULTIPLICATIVELY CONVEX ALGEBRAS. Locally multiplicatively convex (or LMC) algebras are studied in the monograph [8]. We adopt the convention that an LMC algebra is required to be complete and to have an identity. Write $X_{A}$ for the character spare, $\Sigma_{A}$ for the carrier space (set of continuous characters) of an LMC algebra $A$.

Propostrion. Let $\boldsymbol{A}$ be an LMC algebra. Then there exists a bound structure with respect to which $A$ is a psendo-Banach algebra if and only if $X_{A}$ is compact. If $\Sigma_{A}$ is compact, then $X_{A}$ is compact, and if $A$ is also Fréchet, then $X_{A}=\Sigma_{A}$.

Proof. If $A$ is pseudo-Banach, then $X_{A}$ is compact by Corollary 1.4.
Conversely, suppose that $X_{\mathcal{A}}$ is compact, and let $\mathscr{B}$ be the collection of subsets of $A$ described in Example 2.2. Then $(A ; \mathscr{B})$ is a complete bound algebra. Since $A$ has continuous inversion, ([8], Proposition 2.8) it is sufficient, after [1], Corollary 4.2, to show that every element $a$ has a bounded spectrum $\sigma(a)$. But $\sigma(a)=\hat{a}\left(X_{A}\right)$, which is bounded when $X_{A}$ is compact, and so $A$ is pseudo-Banach.

By [8], Corollary 5.6, it is also true that $\sigma\left(a_{t}\right)=\hat{a}\left(\Sigma_{A}\right)$, so that, if $\Sigma_{A}$ is compact, $A$ is pseudo-Banach and $X_{A}$ is compact. (Aliter, since $\hat{a}\left(X_{A}\right)=\hat{a}\left(\Sigma_{A}\right)$ for $a \in A$, every character on $A$ is continuous with respect to the uniform norm $\|\cdot\|_{\infty}$ on $\Sigma_{A}$, and so $X_{A}$ is identified with the compact carrier space of the Banach algebra obtained by completing the algebra of Gelfand transforms of elements of $A$ in the norm $\|\cdot\|_{\infty}$. )

If $A$ is a Fréchet algebra and $\Sigma_{A}$ is compact, then the topology of $A$ is stronger than or equal to that of uniform convergence on $\Sigma_{A}$ ([8], Proposition 8.2), and if $x \in X_{A} \backslash \Sigma_{A}$, then there exists $a \in A$ with $|\hat{a}(x)|$ $>\|\hat{a}\|_{\infty}$, a contradiction of the fact that $\hat{a}\left(X_{A}\right)=\hat{a}\left(\Sigma_{A}\right)$. Thus, $X_{A}=\Sigma_{A}$.

This concludes the proof of the proposition.

Proposition. If $A$ is a Fréchet algebra, then the following are equivalent:

(i) $A$ is a pseudo-Banach algebra;

(ii) $A$ is a Q-algebra - the set of invertible elements is open;

(iii) $\Sigma_{A}$ is compart;

(iv) every element of $A$ has bounded spectrum.

Proof. See [8], Theorem 13.6, and use the above proposition.

EXAMPle 2.4. $p$-Banach Algebras. For the theory of $p$-Banach algebras, see [13]. We use the term ' $p$-Banach' rather than " $p$-normed' to stress that the algebra is complete. Note that the topology of a $p$-Banach algebra is not locally convex in general.

A particular example of a $p$-Banach algebra is the algebra $L^{p}(\boldsymbol{Z})$ with convolution multiplication, together with the $p$-norm $\|x\|=\Sigma\left|x_{n}\right|^{p}$ $(0<p \leqslant 1)$.

We shall describe a bound structure in a commutative $p$-Banach algebra with identity e which makes it a pseudo-Banach algebra.

Let $A$ be a $p$-Banach algebra $(0<p \leqslant 1)$. Suppose that $a_{1}, \ldots, a_{n}$ are elements of $A$ with $0<\left\|a_{i}\right\|<1(i=1, \ldots, n)$. Let $B\left(a_{1}, \ldots, a_{n}\right)$ $=\Delta\left\{a_{1}^{i_{1}} \ldots a_{n}^{i_{n}}: i_{1}, \ldots, i_{n} \geqslant 0\right\}$, the absolutely convex combinations of monomials in $a_{1}, \ldots, a_{n}$ (where $a_{i}^{0}=e$ ).

Propostrion. Let $\mathscr{B}$ consist of the collection of the closures of the sets $B\left(a_{1}, \ldots, a_{n}\right), 0<\left\|a_{i}\right\|<1, i=1, \ldots, n, n=1,2, \ldots$ Then $(A, \mathscr{B})$ is a pseudo-Banach algebra. 
Proof. If $\bar{B} \epsilon \mathscr{B}$, then $\bar{B}$ is absolutely convex, $\bar{B}^{2} \subset \bar{B}$, and $e \epsilon \bar{B}$ Also, $\bar{B}\left(a_{1}, \ldots, a_{m}\right) \cup \bar{B}\left(b_{1}, \ldots, b_{n}\right) \subset \bar{B}\left(a_{1}, \ldots, a_{m}, b_{1}, \ldots, b_{n}\right)$, so that $(A ; \mathscr{B})$ is a bound algebra.

We prove that $B \equiv B\left(a_{1}, \ldots, a_{n}\right)$, and hence $\bar{B}$, is bounded. Choose $r<1$ such that $\left\|a_{i}\right\|<r(i=1, \ldots, n)$. A member $b$ of $B$ can be represented as $b=\Sigma \lambda\left(i_{1}, \ldots, i_{n}\right) a_{1}^{i_{1}} \ldots a_{n}^{i_{n}}$, the sum over $n$-tuples of non-negative integers $\left(i_{1}, \ldots, i_{n}\right)$, at most finitely many of the $\lambda\left(i_{1}, \ldots, i_{n}\right)$ nonzero, and $\Sigma\left|\lambda\left(i_{1}, \ldots, i_{n}\right)\right| \leqslant 1$. Then

$$
\begin{aligned}
\|b\| & \leqslant \sum\left|\lambda\left(i_{1}, \ldots, i_{n}\right)\right|^{p}\left\|a_{1}\right\|^{i_{1}} \ldots\left\|a_{n}\right\|^{i_{n}} \\
& \leqslant\left(\sum_{i_{1}=0}^{\infty}\left\|a_{1}\right\|^{i_{1}}\right) \ldots\left(\sum_{i_{n}=0}^{\infty}\left\|a_{n}\right\|^{i_{n}}\right) \leqslant(1-r)^{-n},
\end{aligned}
$$

so that $B$ is bounded. Thus, the Minkowski functional of $\bar{B}$ is a norm on $A(\bar{B})$.

Since each $\bar{B}$ in $\mathscr{B}$ is bounded, the $\|\cdot\|_{\bar{B}}$ - topology in $A(\bar{B})$ is stronger than the relative topology from $A$. Also, $\bar{B}$ is complete in $A$. Thus, $A(\bar{B})$ is a Banach algebra ([3], I, Proposition 8).

For an $a$ in $A$, choose $\lambda>0$ such that $\|\lambda a\|<1$. Then $a$ belongs to $A(\bar{B}(\lambda a))$.

Thus, $(A ; \mathscr{B})$ is a pseudo-Banach algebra as required.

Many of the properties of $p$-Banach algebras proved by Zielazko follow from their representation as pseudo-Banach algebras.

EXAMPLE 2.5. $A$-HoLOMORPHIC FUNCTIONS. Let $A$ be a natural uniform algebra on the compact space $X$, and let \|\|$_{\infty}$ be the uniform norm on $X$. A continuous function $f$ on $X$ is A-holomorphic on $X$ if, for each $x$ in $X, f$ can be approximated uniformly on some fixed neighbourhood of $x$ in $X$ by functions in $A$ ([5], III, 9). Write $H$ for the algebra of $A$ holomorphic functions on $X$. In general, $H$ is not uniformly closed, [9]. We shall show that $H$ is a natural pseudo-Banach algebra on $X$.

Let $\mathscr{U}=\left\{U_{i}\right\}$ be a (finite) open cover of $X$, and let $H_{\mathscr{U}}=\{f \in C(X)$ : $\left.f \mid U_{j} \in{\overline{A \mid U_{j}}}_{j}, U_{j} \in \mathscr{U}\right\}$, so that $H_{\mathscr{U}}$ (with the uniform norm) is a uniform algebra on $X$. If $\mathscr{U}_{1}$ and $\mathscr{U}_{2}$ are open covers of $X$, let $\mathscr{U}_{12}=\left\{U_{1} \cap U_{2}\right.$ : $\left.U_{i} \in \mathscr{U}_{i}\right\}$, so that $\mathscr{U}_{12}$ is an open cover of $X$. If $B_{\mathscr{Q}}$ is the closed unit ball of $H_{\mathscr{U}}$ and if $\mathscr{B}=\left\{B_{\mathscr{U}}\right\}$, then (i) $B$ is absolutely convex, $B^{2} \subset B, l_{\in} B(B \in \mathscr{B})$, and (ii) $B_{\mathscr{L}_{1}} \cup B_{\mathscr{U}_{2}} \subset B_{\mathscr{U}_{12}}$. Thus, $(H ; \mathscr{B})$ is a complete bound algebra). Since $H=\bigcup H_{\mathscr{L}}, H$ is a pseudo-Banach algebra. By [9], each of the algebras $H_{\mathscr{U}}$ is natural, and so $H$ is a natural pseudo-Banach function algebra.

ExAMPLE 2.6. Germs of ANALYTIC FunCTIONs. If $U$ is an open set in $C^{n}$, write $\mathcal{O}(U)$ for the algebra of functions analytic on $U$, and $H^{\infty}(U)$ for the algebra of functions analytic and bounded on $U$. With the compact-open toplogy, $\mathcal{O}(U)$ is a Fréchet algebra, and $H^{\infty}(U)$ is a Banach algebra with respect to the uniform norm on $U$.

Let $K$ be a compact set in $C^{n}$, and write $\mathcal{O}_{K}$ for the algebra of germs on $K$ of functions analytic in neighbourhoods of $K$. Then $\left\{\mathcal{O}(U) ; r_{V U}\right.$ : $U, \nabla$ open neighbourhoods of $K, V \subset U\}$ is an inductive system of Fréchet algebras and continuous homomorphisms, where $r_{V U}: \mathcal{O}(U) \rightarrow \mathcal{O}(\nabla)$ is the restriction map. We can identify $\mathcal{O}_{K}$ algebraically with the inductive limit of this system, and we give $\mathcal{O}_{K}$ the locally convex inductive limit topology determined by the spaces $\mathcal{O}(U)$. This is the inductive compactopen topology.

We give an explicit representation of $\mathcal{O}_{K}$ as a pseudo-Banach algebra. Let $\mathscr{U}$ be the set of open neighbourhoods $U$ of $K$ such that $\bar{U}$ is compact and each component of $U$ meets $K$. Then $\left\{H^{\infty}(U) ; r_{V U}: U, V \in \mathscr{U}, V \subset U\right\}$ is an inductive system of Banach algebras with identity and continuous unital monomorphisms whose inductive limit is $\mathcal{O}_{K}$. If we write $B_{U}$ for the closed unit ball of $H^{\infty}(U)$, then, by Proposition 1.2, the set $\left\{B_{U}: U \in \mathscr{U}\right\}$ is a bound structure in $\mathcal{O}_{K}$ with respect to which $\mathcal{O}_{K}$ is a pseudo-Banach algebra. We shall call this the standard bound structure in $\mathcal{O}_{K}$.

Note that each of the Banach algebras $H^{\infty}(U)$ is semi-simple, but that $\mathcal{O}_{K}$ is not always semi-simple.

We detail certain properties of $\mathcal{O}_{K}$ which we shall require.

Propositron. The algebra $\mathcal{O}_{K}$ with the inductive compact-open topology has the following properties:

(i) $\mathcal{O}_{K}$ is Hausdorff and fully complete;

(ii) the topology of $\mathcal{O}_{K}$ is the strongest topology, independent of linear structure, with respect to which each of the maps $r_{U}: \mathcal{O}(U) \rightarrow \mathcal{O}_{K}(U$ an open neighbourhood of $K$ ) is continuous;

(iii) $\mathcal{O}_{K}$ is an LMC algebra.

Pro of. First note that, by choosing a countable base $\left\{U_{n}\right\}$ of neighbourhoods of $K$ such that $\bar{U}_{n}$ is compact, $U_{n} \supset \bar{U}_{n+1}$, and each component of $U_{n}$ meets $K$ for all $n$, we may obtain $\mathcal{O}_{K}$ both algebraically and topologically as the inductive limit of an inductive sequence $\left\{\mathcal{O}\left(U_{m}\right) ; r_{n m}: m, n\right.$ $=1,2, \ldots, m \leqslant n\}$ of locally convex spaces and restriction maps $r_{n m}$ which, by the properties of the sequence $\left\{U_{m}\right\}$, are compact linear monomorphisms. Properties (i) and (ii) now follow from general results of Komatsu [7]. 156.

Property (iii) is given by an argument of Waelbroeck, [11], p.

This concludes the proof.

3. The functional calculus. Our first aim in this section is to establish the analytic functional calculus for pseudo-Banach algebras, 
Let $A$ be a commutative algebra with identity $e$. For $a_{1}, \ldots, a_{n}$ in $A$, let $\mathrm{id}_{A}\left(a_{1}, \ldots, a_{n}\right)$ denote the ideal generated in $\mathrm{A}$ by $a_{1}, \ldots, a_{n}$. We write $A^{n}=\left\{\boldsymbol{a}=\left(a_{1}, \ldots, a_{n}\right): a_{i} \in A\right\}$ and $A^{\infty}=\bigcup\left\{A^{n}: n=1,2, \ldots\right\}$. If $\boldsymbol{a}$ belongs to $A^{n}$, then the joint spectrum of $\boldsymbol{a}$ in $A, \sigma_{A}(\boldsymbol{a})$ or $\sigma(\boldsymbol{a})$, is given by

$$
\begin{aligned}
\sigma_{A}(\boldsymbol{a}) & =\sigma_{A}\left(a_{1}, \ldots, a_{n}\right) \\
& =\left\{\left(\lambda_{1}, \ldots, \lambda_{n}\right) \epsilon C^{n}: \operatorname{id}_{A}\left(a_{1}-\lambda_{1} e, \ldots, a_{n}-\lambda_{n} e\right) \text { is proper }\right\} .
\end{aligned}
$$

If $A$ is a pseudo-Banach algebra with character space $X_{A}$, then it is an immediate consequence of Proposition 1.5 that $\sigma_{A}(\boldsymbol{a})=\hat{\boldsymbol{a}}\left(X_{A}\right)$, and so $\sigma_{A}(\boldsymbol{a})$ is a non-empty compact set in $\boldsymbol{C}^{n}$.

Write $\mathcal{O}[\sigma(\boldsymbol{a})]$ for the algebra of function germs $\mathcal{O}_{\sigma(\boldsymbol{a})}$. Algebras of germs of analytic functions always have the inductive compact-open topology described in Example 2.6

Denote a general point of $C^{n}$ by $z=\left(z_{1}, \ldots, z_{n}\right)$. For $n \geqslant m$, the projection map $p_{m n}: \boldsymbol{C}^{n} \rightarrow \boldsymbol{C}^{m}$ defined by $p_{m n}\left(z_{1}, \ldots, z_{n}\right)=\left(z_{1}, \ldots, z_{m}\right)$ is continuous and open. Define a dual map $q_{m n}$ to $p_{m n}$ by $q_{m n}(f)$ $=f \circ p_{m n}(f \in \mathcal{O}(U))$ for any open subset $U$ of $C^{m}$, so that $q_{m n}: \mathcal{O}(U) \rightarrow$ $\mathcal{O}\left(p_{m n}{ }^{-1},(U)\right)$ is a continuous monomorphism.

If $n \geqslant m$, if $\boldsymbol{a}^{\prime}=\left(a_{1}, \ldots, \boldsymbol{a}_{m}, \ldots, \boldsymbol{a}_{n}\right)$, and if $\boldsymbol{a}=\left(a_{1}, \ldots, \boldsymbol{a}_{m}\right)$, then $p_{m n}\left(\sigma\left(\boldsymbol{a}^{\prime}\right)\right)=\sigma(\boldsymbol{a})$, and therefore composition with $p_{m n}$ induces a continuous monomorphism, again denoted by $q_{m n}$, from $\mathcal{O}[\sigma(\boldsymbol{a})]$ into $\mathcal{O}\left[\sigma\left(\boldsymbol{a}^{\prime}\right)\right]$.

We now give a statement of the existence and uniqueness of the (strong) functional calculus for Banach algebras. The notation is based on that of Bourbaki [4].

THEOREM 3.1. Let A be a commutative Banach algebra with identity $e$. Then there exists a unique map $\boldsymbol{a} \rightarrow \Theta_{\boldsymbol{a}}$ which associates with each $\boldsymbol{a}$ in $A^{\infty}$ a continuous homomorphism $\Theta_{\boldsymbol{a}}: \mathcal{O}[\sigma(\boldsymbol{a})] \rightarrow A$ with the following properties:

(i) if $\boldsymbol{a}=\left(a_{1}, \ldots, \boldsymbol{a}_{m}\right)$, then $\Theta_{\boldsymbol{\alpha}}\left(z_{i}\right)=a_{i}(i=1, \ldots, m)$, and also $\Theta_{\boldsymbol{a}}(1)=e$;

(ii) if $1 \leqslant m \leqslant n$, and if $\boldsymbol{a}^{\prime}=\left(a_{1}, \ldots, a_{m} ; \ldots, a_{n}\right)$, then $\Theta_{\boldsymbol{a}^{\prime}} \circ q_{m n}=\Theta_{\boldsymbol{a}}$. Proofs of this result may be found in [4] and [5].

Let $A$ be a pseudo-Banach algebra, and identify $A$ with the limit of the inductive system $\left\{A_{\alpha} ; \pi_{\beta \alpha}: \alpha, \beta \epsilon \Lambda, \alpha \leqslant \beta\right\}$ of Banach algebras and continuous monomorphisms as in Proposition 1.2. We may regard the $A_{\alpha}$ as subalgebras of $A$.

If $\boldsymbol{a} \in A_{a}^{\infty}$, let $\sigma_{a}(\boldsymbol{a})$ denote the joint spectrum of $\boldsymbol{a}$ in $A_{a}$. The next lemma gives the relations between joint spectra with respect to $A$ and those with respect to the $A_{\alpha}$ which we require for the functional calculus.

LEMma 3.2. Let $\boldsymbol{a}=\left(a_{1}, \ldots, a_{n}\right)$ belong to $A^{\infty}$, and let $\Xi=\left\{a: a_{1}, \ldots\right.$ $\left.\ldots, a_{n} \in A_{a}\right\}$. Then (i) $\sigma_{A}(a)=\bigcap\left\{\sigma_{\alpha}(\boldsymbol{a}): \alpha \epsilon \Xi\right\}$,

(ii) if $U$ is any neighbourhood of $\sigma_{A}(a)$ in $C^{n}$, there exists a in $\Xi$ such that $\sigma_{a}(\boldsymbol{a}) \subset U$,

(iii) the algebra $\mathcal{O}\left[\sigma_{A}(\boldsymbol{a})\right]$ is homeomorphically isomorphic with the inductive limit of the system $\left\{\mathcal{O}\left[\sigma_{a}(\boldsymbol{a})\right] ; r_{\beta \alpha}: \alpha, \beta \in \Xi, \alpha \leqslant \beta\right\}$ of LMC algebras and continuous homomorphisms, where $r_{\beta \alpha}$ is the natural 'restriction' map.

Proof. (i) Let the intersection be $S$; clearly, $\sigma_{A}(a) \subset S$. Conversely, if $\left(\lambda_{1}, \ldots, \lambda_{n}\right) \notin \sigma_{A}(\boldsymbol{a})$, then there exist $b_{1}, \ldots, b_{n} \in A$ such that $\sum_{i=1}^{n}\left(a_{i}-\right.$ $\left.-\lambda_{i} e\right) b_{i}=e$. Choose $\alpha$ such that $a_{1}, \ldots, a_{n}, b_{1}, \ldots, b_{n} \in A_{a} ;\left(\lambda_{1}, \ldots, \lambda_{n}\right)$ $\notin \sigma_{a}(\boldsymbol{a})$, so that $\mathrm{S} \subset \sigma_{A}(\boldsymbol{a})$, as required.

(ii) For each $\alpha \epsilon \Xi, \sigma_{\alpha}(\boldsymbol{a}) \backslash U$ is a compact set in $\boldsymbol{C}^{n}$, and, by (i), $\bigcap\left\{\sigma_{\alpha}(\boldsymbol{a}) \backslash U: \alpha \in \Xi\right\}=\varnothing$. By the finite intersection property, some finite set of the $\sigma_{\alpha}(\boldsymbol{a}) \backslash U$ has null intersection, and from the directedness of $\Lambda$ follows the existence of $\alpha$ in $\Xi$ with $\sigma_{\alpha}(\boldsymbol{a}) \backslash U=\varnothing$.

(iii) If $\alpha, \beta \in \Xi$ with $\alpha \leqslant \beta$, then $\sigma_{\alpha}(\boldsymbol{a}) \supset \sigma_{\beta}(\boldsymbol{a})$. Thus each germ $\boldsymbol{f}$ in $\mathcal{O}\left[\sigma_{\alpha}(a)\right]$ determines a corresponding germ $r_{\beta \alpha}(\boldsymbol{f})$ in $\mathcal{O}\left[\sigma_{\beta}(\boldsymbol{a})\right]$, and $r_{\beta a}$ is a continuous homomorphism from $\mathcal{O}\left[\sigma_{\alpha}(\boldsymbol{a})\right]$ to $\mathcal{O}\left[\sigma_{\beta}(\boldsymbol{a})\right]$. Since $\Xi$ is directed upwards by $\leqslant$, it follows that $\left\{\mathcal{O}\left[\sigma_{\alpha}(\boldsymbol{a})\right] ; r_{\beta \alpha}: \alpha, \beta \epsilon \Xi, \alpha \leqslant \beta\right\}$ is an inductive limit system. Since any function analytic in an open neighbourhood of $\sigma_{A}(\boldsymbol{a})$ is, by (ii), analytic in a neighbourhood of some $\sigma_{\alpha}(\boldsymbol{a})$, it follows that the inductive limit of the system $\left\{\mathcal{O}\left[\sigma_{\alpha}(\boldsymbol{a})\right] ; r_{\beta \alpha}\right\}$ is isomorphic with $\mathcal{O}\left[\sigma_{A}(\boldsymbol{a})\right]$. That the inductive compact-open topology of $\mathcal{O}\left[\sigma_{A}(a)\right]$ coincides with the locally convex inductive limit topology determined by the algebras $\mathcal{O}\left[\sigma_{a}(\boldsymbol{a})\right](\alpha \in \Xi)$ is straightforward to verify.

The lemma is proved.

In view of (3.2) (iii), we identify $\mathcal{O}\left[\sigma_{A}(\boldsymbol{a})\right]$ with the inductive limit of the $\mathcal{O}\left[\sigma_{\alpha}(a)\right](\alpha \in \Xi)$. When this is done, the natural map $r_{\alpha}$ from $\mathcal{O}\left[\sigma_{\alpha}(a)\right]$ into the inductive limit is just the operation of 'restricting' germs from $\sigma_{\alpha}(\boldsymbol{a})$ to $\sigma_{A}(\boldsymbol{a})$.

THEOREM 3.3. Let $(A, \mathscr{B})$ be a pseudo-Banach algebra. Then there exists a unique map $\boldsymbol{a} \rightarrow \Theta_{\boldsymbol{a}}$ which associates with each $\boldsymbol{a}$ in $A^{\infty}$ a homomorphism $\Theta_{\boldsymbol{a}}: \mathcal{O}\left[\sigma_{A}(\boldsymbol{a})\right] \rightarrow A$ with the following properties:

(i) if $\boldsymbol{a}=\left(a_{1}, \ldots, a_{m}\right)$, then $\Theta_{\boldsymbol{a}}\left(z_{i}\right)=a_{i}(i=1, \ldots, m)$, and also $\Theta_{\boldsymbol{a}}(1)=e$

(ii) if $1 \leqslant m \leqslant n$, and $\boldsymbol{a}^{\prime}=\left(a_{1}, \ldots, a_{m}, \ldots, a_{n}\right)$, then $\Theta_{\boldsymbol{a}} \circ q_{m n}=\Theta_{\boldsymbol{a}}$;

(iii) for each $\alpha$ such that $a_{1}, \ldots, a_{m}$ are in $A_{\alpha}, \Theta_{a}\left(r_{\alpha}\left(\mathcal{O}\left[\sigma_{\alpha}(\boldsymbol{a})\right]\right)\right) \subset A_{\alpha}$ and $\Theta_{\boldsymbol{a}} \circ \boldsymbol{r}_{\alpha}: \mathcal{O}\left[\sigma_{\alpha}(\boldsymbol{a})\right] \rightarrow A_{\alpha}$ is continuous:

Proof. First we clarify certain abuses of notation. For each $\alpha$, there is a collection of maps $r_{a}: \mathcal{O}\left[\sigma_{\alpha}(\boldsymbol{a})\right] \rightarrow \mathcal{O}\left[\sigma_{\mathcal{A}}(\boldsymbol{a})\right]$, one for each $\boldsymbol{a} \in A_{a}^{\infty}$, and we are using symbol $r_{a}$ to denote any of these. Similarly, if $\boldsymbol{a}$ and $\boldsymbol{a}^{\prime}$ 
are as in (ii) and $\alpha$ is such that $a_{1}, \ldots, a_{n} \in A_{a}$, the projection $p_{m n}$ induces homomorphisms $\mathcal{O}\left[\sigma_{A}(\boldsymbol{a})\right] \rightarrow \mathcal{O}\left[\sigma_{A}\left(\boldsymbol{a}^{\prime}\right)\right]$ and $\mathcal{O}\left[\sigma_{a}(\boldsymbol{a})\right] \rightarrow \mathcal{O}\left[\sigma_{a}\left(\boldsymbol{a}^{\prime}\right)\right]$, and we write $q_{m n}$ for any of these homomorphisms. With these conventions, the following diagram commutes

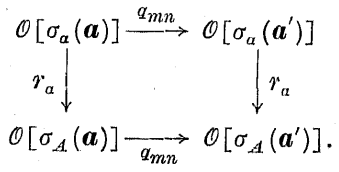

So, if maps $\Theta_{\boldsymbol{a}}$ with the properties (i), (ii), (iii) exist, then, for fixed $\alpha$ in $\Lambda$, the maps $\Theta_{a} \circ r_{\alpha}\left(\boldsymbol{a} \epsilon A_{a}^{\infty}\right)$ form a collection of homomorphisms satisfying the conditions of Theorem 3.1 for the Banach algebra $A_{\alpha}$, and the $\Theta_{\boldsymbol{a}} \circ r_{\alpha}$ must be the unique continuous functional calculus homomorphisms for $A_{a}$. Thus the uniqueness of the $\Theta_{a}$ follows from their existence, and to prove the existence it is clearly sufficient to prove that the Banach algebra functional calculi for the different $A_{\alpha}$ can be superimposed to give a welldefined functional calculus homomorphism for $A$. Precisely, if we write $\Theta_{a}^{a}$ for the functional calculus homomorphism for $A_{\alpha}\left(\boldsymbol{a} \in A_{\alpha}^{\infty}\right)$, we must prove that $\Theta_{a}^{\beta} \circ r_{\beta a}=\pi_{\beta a} \circ \Theta_{a}^{\alpha}$ for $\alpha \leqslant \beta$. But this is an immediate consequence of the continuity of the $\Theta_{a}^{\beta} \circ r_{\beta \alpha}$ and the $\pi_{\beta \alpha} \circ \Theta_{a}^{\alpha}$, the fact that they coincide on polynomials, and polynomial approximation with the technique of Arens and Calderón [2].

The theorem is proved.

As a corollary, we state the weak form of the functional calculus. It is the weak form which is required for many of the applications.

CoRollary 3.4. Let $\boldsymbol{a}=\left(a_{1}, \ldots, a_{n}\right)$ belong to $\mathrm{A}^{n}$, and suppose that $f$ is a function which is analytic on some neighbourhood of $\sigma_{A}(\boldsymbol{a})$ in $\boldsymbol{C}^{n}$. Then there exists an element $g$ in A such that

$$
\hat{g}(x)=f \circ\left(\hat{a}_{1}(x), \ldots, \hat{a}_{n}(x)\right) \quad\left(x \in X_{A}\right) .
$$

We shall also require the following result, which is a straightforward corollary of the corresponding result for Banach algebras.

Propositron 3.5. Given $\boldsymbol{a}$ in $\mathrm{A}^{\infty}$ and $\boldsymbol{f}_{1}, \ldots, \boldsymbol{f}_{\boldsymbol{n}}$ in $\mathcal{O}\left[\sigma_{A}(\boldsymbol{a})\right]$, let $b_{\text {. }}$ $=\Theta_{\boldsymbol{a}}\left(\boldsymbol{f}_{\boldsymbol{i}}\right)(\boldsymbol{i}=1, \ldots, n)$, and let $\boldsymbol{b}=\left(b_{1}, \ldots, b_{n}\right)$. Then, for any $\boldsymbol{F}$ in $\mathcal{O}\left[\sigma_{A}(\boldsymbol{b})\right]$

$$
\Theta_{b}(\boldsymbol{F})=\Theta_{a}\left(\boldsymbol{F} \circ\left(f_{1}, \ldots, f_{n}\right)\right) .
$$

As we pointed out in (2.6), the algebra $\mathcal{O}_{K}$ of germs of analytic functions on a compact set $K$ in $\boldsymbol{C}^{n}$ is a pseudo-Banach algebra with respect to a standard bound structure, say $\mathscr{S}$, consisting of the unit balls $B_{U}$ of the algebras $H^{\infty}(U)$ for $U$ belonging to $\mathscr{U}(K)$, the collection of open neighbourhoods of $K$ which have compact closures and each component of which meets $K$. We show that for a given pseudo-Banach algebra, the functional calculus homomorphisms of Theorem (3.3) are boundpreserving (Definition 1.8), and that the homomorphisms are characterized by the property of being bound-preserving, together with the usual algebraic properties. Theorem 3.6 below is perhaps a more natural statement than Theorem 3.3.

THEOREM 3.6. Let $(A ; \mathscr{B})$ be a pseudo-Banach algebra. Then there exists a unique map $\boldsymbol{a} \rightarrow \Theta_{\boldsymbol{a}}$ which associates with each $\boldsymbol{a}$ in $A^{\infty}$ a boundpreserving homomorphism $\Theta_{\boldsymbol{a}}: \mathcal{O}\left[\sigma_{A}(\boldsymbol{a})\right] ; \mathscr{S} \rightarrow(A ; \mathscr{B})$ wich satisfies (i) and (ii) of Theorem 3.3 .

Proof. Let $\boldsymbol{a} \in \boldsymbol{A}^{\infty}$. To show that the map $\Theta_{\boldsymbol{a}}$ constructed in Theorem 3.3 is bound-preserving, it is clearly sufficient to prove that, given $U \epsilon \mathscr{U}\left(\sigma_{A}(a)\right)$, there exists $a$ such that $\Theta_{\boldsymbol{a}}\left(H^{\infty}(U)\right) \subset A_{a}$ and $\Theta_{\boldsymbol{a}} \mid H^{\infty}(U)$ : $H^{\infty}(U) \rightarrow A_{\alpha}$ is continuous. But this is immediate if, as we may, we choose $\alpha$ such that $a \in A_{\alpha}^{\infty}$ and $\sigma_{\alpha}(\boldsymbol{a}) \subset U$.

To prove the uniqueness, let $\boldsymbol{a} \rightarrow \Phi_{\boldsymbol{a}}$ be any map satisfying the given conditions. Fix $\boldsymbol{a}=\left(a_{1}, \ldots, a_{m}\right)$ in $A^{\infty}$ and $U$ in $\mathscr{U}\left(\sigma_{A}(\boldsymbol{a})\right)$, and let $\mathscr{B}$ $=\left\{B_{a}: \alpha \in \Lambda\right\}$ be the bound structure in $A$. Since $\Theta_{\boldsymbol{a}}$ and $\Phi_{\boldsymbol{a}}$ are both bound-preserving, we can choose $\alpha, \beta$ in $\Lambda$ and $s, t>0$ such that $\Theta_{a}\left(B_{U}\right)$ $\subset s B_{a}$ and $\Phi_{a}\left(B_{U}\right) \subset t B_{\beta}$. Choose $\gamma$ in $\Lambda$ such that $a \leqslant \gamma, \beta \leqslant \gamma$, and $\sigma_{\gamma}(\boldsymbol{a}) \subset U$, and ahoose $\lambda>0$ such that $B_{\alpha} \cup B_{\beta} \subset \lambda B_{\gamma}$. If $r=\max \{\lambda s, \lambda t\}$, then $\Theta_{a}\left(B_{U}\right) \subset r B$ and $\Phi_{a}\left(B_{U}\right) \subset r B$, so both $\Theta_{a}$ and $\Phi_{a}$ when restricted to $H^{\infty}(U)$ give continuous homomorphisms into $A_{\gamma}$.

Now fix $f$ in $H^{\circ}(U)$. Using the technique of Arens and Calderón [2] we can choose elements $a_{m+1}, \ldots, a_{n}$ in $A$ and a neighbourhood $V$ of $\sigma_{\gamma}\left(\boldsymbol{a}^{\prime}\right)$ in $O^{n}\left(\right.$ where $\left.\boldsymbol{a}^{\prime}=\left(a_{1}, \ldots, a_{n}\right)\right)$ such that $q_{m n}(f)$ is in $H^{\infty}(V)$ and is the uniform limit on $V$ of a sequence of polynomials in $n$ variables. If necessary, take $\gamma$ further along the directed set $\Lambda$ so that $\Theta_{a^{\prime}}$ and $\Phi_{a^{\prime \prime}}$ restriced to $H^{\infty}(V)$, give continuous homomorphisms into $A_{\gamma}$. By condition (i), $\Theta_{\alpha}$ and $\Phi_{\alpha}$ coincide on polynomials, and then, using (ii) and the continuity, we have $\Theta_{\boldsymbol{a}}(f)=\Theta_{\boldsymbol{a}^{\prime}}\left(q_{m n}(f)\right)=\Phi_{\boldsymbol{a}^{\prime}}\left(q_{m n}(f)\right)=\Phi_{\boldsymbol{a}}(f)$. Since $\quad U$ in $\left(\sigma_{A}(a)\right)$ and $f$ in $H^{\circ}(U)$ were arbitrary, this proves that $\Theta_{\boldsymbol{a}}=\Phi_{\boldsymbol{a}}$ for any $\boldsymbol{a}$ in $A^{\infty}$, and completes the proof of the theorem.

All the examples of pseudo-Banach algebras which we considered in $\S 2$ are also topological algebras. It is therefore of interest to investigate the continuity of the pseudo-Banach functional calculus with respect to the topologies of these examples.

THEOREM 3.7. Let $(A ; \mathscr{B})$ be a pseudo-Banach algebra which is also a topological algebra for the topology $\tau$. Suppose each member of $\mathscr{B}$ is $\tau$-bounded. Then the functional calculus homomorphisms of Theorem (3.3) are continuous with respect to $\tau$.

Proof. Let $\boldsymbol{a} \in A^{\infty}$. By (2.6) (ii) it is sufficient to prove that $\Theta_{\boldsymbol{a}} \circ \boldsymbol{r}_{U}$ : $\mathcal{O}(U) \rightarrow A$ is continuous for each open neighbourhood $U$ of $\sigma_{A}(a)$. Given Studia Mathematica XL.1 
$\mathrm{U}$, choose $a$ such that $\boldsymbol{a} \in A_{\alpha}^{\infty}$ and $\sigma_{a}(\boldsymbol{a}) \subset U$. There is a homomorphism (formation of germs) $r_{U}^{a}: \mathcal{O}(U) \rightarrow \mathcal{O}\left[\sigma_{a}(\boldsymbol{a})\right]$ such that $\Theta_{a} \circ r_{U}=\Theta_{a} \circ r_{a} \circ r_{U}^{a}$ and it follows that $\Theta_{a} \circ r_{U}$ takes values in $A_{a}$ and is continnous with respect to the $\|\cdot\|_{\alpha}$-topology on $A_{\alpha}$. But since $B_{a}$ is $\tau$-bounded in $A$, the $\|\cdot\|_{a^{-}}$ topology is no weaker than the relative topology induced by $\tau$ on $A_{a}$, and so $\Theta_{\boldsymbol{a}} \circ r_{U}$ is continuous with respect to $\tau$, and the theorem is proved. Note that the hypotheses of this theorem include all the examples of $\S 2$.

The existence of a continuous functional calculus for $p$-Banach algebras is due to Gramsch [6]. As such a calculus is necessarily unique, Theorem (3.3) provides an alternative method of constructing the calculus which, given the result for Banach algebras, may be easier than the constructions involving integrals used in [6].

We now show that the existence of an analytic functional calculus essentially characterizes pseudo-Banach algebras. We consider only the semi-simple case, where the result is most naturally formulated.

Let $X$ be a compact Hausdorff space, and let $A$ be a function algebra on $X$. Given $\boldsymbol{a}$ in $A^{\infty}$ and $f$ in $\mathcal{O}[\boldsymbol{a}(X)], \Theta_{\boldsymbol{a}}[\boldsymbol{f})$ is the function in $O(X)$ defined by $\Theta_{\boldsymbol{a}}(\boldsymbol{f})(x)=(f \circ a)(x)(x \in X)$. We say that analytic functions operate on $\mathrm{A}$ if $\Theta_{\boldsymbol{a}}(\mathcal{O}[\boldsymbol{a}(X)]) \subset A$ for all $\boldsymbol{a}$ in $A^{\infty}$.

THEOREM 3.8. Let $A$ be a function algebra on $X$. If analytic functions operate on $A$, then there exists a bound structure in $A$ with respect to which $A$ is a pseudo-Banach algebra.

Proof. For $\boldsymbol{a} \in A^{\infty}$, let $A_{a}=\Theta_{a}(\mathcal{O}[\boldsymbol{a}(X)])$, with the quotient topology from $\mathcal{O}[\boldsymbol{a}(X)]$. The kernel of $\Theta_{\boldsymbol{a}}$ is $\{\boldsymbol{f} \in \mathcal{O}[\boldsymbol{a}(X)]: \boldsymbol{f}(\boldsymbol{a}(X))=\{0\}\}$, and is therefore a closed ideal in $\mathcal{O}[\boldsymbol{a}(X)]$. Since $\mathcal{O}[\boldsymbol{a}(X)]$ is an LMC algebra, Example 2.6 (iii), so is $A_{\boldsymbol{a}}$ ([8], Proposition 2.4(e)), and since $\mathcal{O}[\boldsymbol{a}(X)]$ is fully complete (Example 2.6 (i)), so is $A_{a}$ ([10], p. 114). Thus, $A_{a}$ is a (complete) LMC algebra. Since $\mathcal{O}[\boldsymbol{a}(X)]$ has compact character space, so has $A_{\boldsymbol{a}}$, and $A_{\boldsymbol{a}}$ is a pseudo-Banach algebra with respect to the bound structure $\mathscr{B}_{a}$ given in Example 2.2. Let $\mathscr{B}=\bigcup\left\{\mathscr{B}_{\boldsymbol{a}}: a \in A^{\infty}\right\}$. Then we claim that $\mathscr{B}$ is a bound structure with respect to which $A$ is a psendoBanach algebra.

We verify condition (ii) of Definition 1.1. Let $B_{1}, B_{2}$ be in $\mathscr{B}$, say $B_{1} \in \mathscr{B}_{\boldsymbol{a}}, B_{2} \in \mathscr{B}_{\boldsymbol{b}}$, where $\boldsymbol{a}=\left(a_{1}, \ldots, a_{m}\right)$ and $\boldsymbol{b}=\left(b_{1}, \ldots, b_{n}\right)$. Set $\boldsymbol{c}=\left(a_{1}, \ldots\right.$ $\left.\ldots, a_{m}, b_{1}, \ldots, b_{n}\right)$, and let $p_{1}: \boldsymbol{C}^{m+n} \rightarrow \boldsymbol{C}^{m}, p_{2}: \boldsymbol{C}^{m+n} \rightarrow C^{n}$ be the projections onto the first $m$ and the last $n$ coordinates, respectively. Then $p_{1}(\boldsymbol{c}(X))=\boldsymbol{a}(X), p_{2}(\boldsymbol{c}(X))=\boldsymbol{b}(X)$, and the following diagrams commute. In each case, the map $i$ is inclusion.
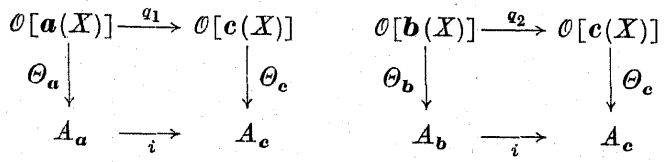

Since $A_{a}$ and $A_{b}$ have the quotient topologies, the inclusion maps are continuous, and therefore $B_{1}$ and $B_{2}$ are bounded in $A_{c}$. Then $B_{1} B_{2}$ is bounded in $A_{c}$, and if $B_{3}$ is the closture in $A_{c}$ of the set $\Delta\left(B_{1} \cup B_{2} \cup B_{1} B_{2}\right)$, then $B_{3} \in \mathscr{P}$ and $B_{1} \cup B_{2} \subset B_{3}$.

The other conditions are clearly satisfied, and so the theorem is proved.

That a function algebra on which analytic functions operate is not necessarily a natural pseudo-Banach algebra will be shown in Example 4.7.

4. Applications. Let $A$ be a pseudo-Banach algebra with character space $X_{A}$. Then we have identified the maximal ideals of $A$ with the kernels of characters, so that $\sigma_{\mathcal{A}}(\boldsymbol{a})=\hat{\boldsymbol{a}}\left(X_{\mathcal{A}}\right)\left(\boldsymbol{a} \in A^{\infty}\right)$, and we have established the functional calculus for $A$. Using these results, it is possible to establish for pseudo-Banach algebras a number of the standard results for Banach algebras.

THEOREM 4.1 (IMPLICIT FUNCTION THEOREM). Let $a_{1}, \ldots, a_{n}$ belong to the pseudo-Banach algebra $A$. Let $h$ belong to $O\left(X_{A}\right)$, and let $\sigma \equiv\left(h, \hat{a}_{1}, \ldots\right.$ $\left.\ldots, \hat{a}_{n}\right)\left(X_{A}\right)$. Let $F\left(w, z_{1}, \ldots, z_{n}\right)$ be a function analytic in a neighbourhood of $\sigma$ such that $F\left(h, \hat{a}_{1}, \ldots, \hat{a}_{n}\right)=0$ on $\sigma$, while $\partial F / \partial w \neq 0$ on $\sigma$. Then there exists a unique element $g$ in $A$ with $\hat{g}=h$ and $F\left(\hat{g}, \hat{a}_{1}, \ldots, \hat{a}_{n}\right)=0$.

Proof. See [5], III, Theorem 6.1. The properties of $A$ required are those stated above, together with the substitution theorem which we have stated as Proposition 3.5. This latter is required in the proof of both the existence and the uniqueness of $g$.

COROLLART 4.2. If $a$ is an invertible element of $A$, and if there exists $h$ in $C\left(X_{A}\right)$ such that $h^{n}=\hat{a}$, then there is $g$ in $A$ such that $g^{n}=a$.

COROLLARY 4.3 (ŠHOV IDEMPOTENT THEOREM). Let $\boldsymbol{X}_{0}$ be a nonempty open and closed subset of $X_{A}$. Then there is a unique idempotent a in A such that $\hat{a}\left(X_{0}\right)=\{1\}$ and $\hat{a}\left(\bar{X} \backslash X_{0}\right)=\{0\}$.

The remaining results require a form of the Arens-Calderón lemma [2] applicable to pseudo-Banach algebras.

Lemra 4.4. Let $A$ be a pseudo-Banach algebra. If $\boldsymbol{a}=\left(a_{1}, \ldots, a_{m}\right)$ belongs to $A^{m}$, and if $U$ is a neighbourhood of $\sigma_{\mathcal{A}}(\boldsymbol{a})$ in $C^{m}$, then there exist $a_{m+1}, \ldots, a_{n}$ in $A$ and an open polynomial polyhedron $\nabla$ containing $\sigma_{\mathcal{A}}\left(a_{1}, \ldots\right.$ $\left.\ldots, a_{n}\right)$ such that $p(V) \subset U$, where $p$ is the projection of $\boldsymbol{C}^{n}$ onto $\boldsymbol{C}^{m}$.

Proof. Let $A_{1}$ be the uniformly closed subalgebra of $C\left(X_{\Lambda}\right)$ generated by $\hat{a}_{1}, \ldots, \hat{a}_{m}$, and let $\sigma_{1}(\boldsymbol{a})$ be the joint spectrum of $\boldsymbol{a}$ in $A_{1}$, so that $\sigma_{1}(\boldsymbol{a}) \supset \boldsymbol{a}\left(X_{A}\right)=\sigma_{\mathcal{A}}(\boldsymbol{a})$. Suppose that $z \epsilon \sigma_{1}(\boldsymbol{a}) \backslash U$, and let $J=\mathrm{id}_{\mathcal{1}}\left(a_{1}-\right.$ $\left.-z_{1} e, \ldots, a_{m}-z_{m} e\right)$. Then $J$ is not a proper ideal in $A$, and so there exist $a_{m+1}, \ldots, a_{2 m} \in A$ such that $\sum_{i=1}^{m}\left(a_{i}-z_{i} e\right) a_{m+i}=1$. Let $A_{2}$ be the uniformly closed subalgebra of $C\left(X_{A}^{i=1}\right)$ generated by $a_{1}, \ldots, a_{2 m}$, and let $\sigma_{2}(\boldsymbol{a})$ be 
the joint spectrum of $\boldsymbol{a}$ in $A_{2}$. Then $\sigma_{A}(\boldsymbol{a}) \subset \sigma_{2}(\boldsymbol{a}) \subset \sigma_{1}(\boldsymbol{a})$ and $z \notin \sigma_{2}(\boldsymbol{a})$. By a standard compactness argument, we can find $a_{2 m+1}, \ldots$ $\ldots, a_{n} \in A$ such that, if $B$ is the closed subalgebra of $C\left(X_{A}\right)$ generated by $a_{1}, \ldots, a_{12}$, then $\sigma_{A}(\boldsymbol{a}) \subset \sigma_{B}(\boldsymbol{a}) \cdot \subset U$. The result follows from the facts that $\sigma_{B}\left(a_{1}, \ldots, a_{i 2}\right)$ is a compact, polynomially convex subset of $C^{n}$ containing $\left(\hat{a}_{1}, \ldots, \hat{a}_{n}\right)\left(X_{A}\right)=\sigma_{A}\left(a_{1}, \ldots, a_{n}\right)$, and that $p\left(\sigma_{B}\left(a_{1}, \ldots, a_{n}\right)\right)=\sigma_{B}(\boldsymbol{a})$ $\subset U$. The lemma is proved.

If $A$ is pseudo-Baniwch algebra, let $A^{-1}$ denote the (multiplicative group of ) invertible elements of $A$. For $a$ in $A$, and for $a$ such that $a$ belongs to $A_{\alpha}$, the series $\Sigma a^{n} / n$ ! converges in $A_{\alpha}$ to an element exp $a$. It is easy to see that $\exp a$ is independent of $a$ and that the map $a \rightarrow \exp a$ is a homomorphism from the additive group of $A$ to $A^{-1}$. We can now give the following form of the Arens-Royden theorem.

THEOREM 4.5 (ARENS-ROXDEN THEOREM). Let $A$ be a pseudo-Banach algebra. Then $A^{-1} / \exp A$ is isomorphic to $H^{1}\left(X_{A}, \boldsymbol{Z}\right)$.

Proof. See [5], III, 7. In the proof, we require the above lemma and a corollary of the implicit function theorem which states that, if a belongs to $A$, and if $\hat{\mathrm{a}}$ has a continuous logarithm on $X_{A}$, then $a=\exp b$ for some $b$ in $A$.

THEOREM 4.6 (ROSSI'S LOCAL PEAK SET THEOREM). Every local peak set in the character space of a pseudo-Banach algebra is a peak set.

Proof. See [5], III, 8. We again use Lemma 4.4.

In particular, every local peak point in the character space is a peak point.

If $A$ is an LMC algebra, then $A$ has a representation as an algebra of continuous functions on its carrier space $\Sigma_{\mathcal{A}}$, and a functional calculus holds for $A$ defined on $\Sigma_{A}$. It might therefore be conjectured that the local peak point theorem would hold for an LMC algebra with compact carrier space defined on that carrier space. However, the final example, which is a non-natural pseudo-Banach algebra on which analy tic functions operate, shows that this is not so.

EXAMPLE 4.7. A FAILURE OF TIIE LOCAL PEAK POINT THEOREM. Let $\Delta_{r}=\left\{(z, w)_{\epsilon} \boldsymbol{C}^{2}:|z|,|w|<r\right\}$, so that $\Delta_{r}$ is a bicylinder in $\boldsymbol{C}^{2}$. Let $Y$ $=\left(\Delta_{1} \backslash \Delta_{\frac{1}{2}}\right) \cup\left\{(z, w): w=0,\left|z-\frac{1}{2}\right| \leqslant \frac{1}{2}\right\}$, so that $Y$ is a compact subset of $C^{2}$.

A slice of $Y$ is a subset formed by fixing one coordinate, and if $K$ is a slice, int $K$ denotes the interior of $K$ with respect to the complex plane in $C^{2}$ containing it. Let $\mathscr{K}$ be the collection of subsets of $Y$ which are compact and which are a countable union of slices.

For $K \in \mathscr{K}$, let $A_{K}=\{f \in C(K): f \mid$ int $S$ is analytic, for each slice $S$ in $K\}$. Then $A_{K}$ is a uniform algebra on $K$ with carrier space $K$ ([5], II, Theorem 1.9). Let $A=\left\{f \in O(Y): f \mid K \in A_{K}(K \in \mathscr{K})\right\}$, with the topology of uniform convergence on the sets of $\mathscr{K}$. Then it is easy to see that $A$ is a (complete) LMC algebra with respect to the directed family of seminorms $\left\{p_{K}\right\}$, where $p_{K}(f)=\sup |f(x)|(K \in \mathscr{K})$, and that $A=\operatorname{limproj} A_{K}$. Thus, by [8], Proposition $\tau .5, \Sigma_{A}=\bigcup K=Y$.

With the topology of uniform convergence on $Y, A=P(Y)$, and the character space of $A$ is $\bar{\Delta}_{1}$ (c. f. [5], III, 3), a proper superset of $Y$.

The origin is a local peak point for $A$ with respect to the carrier space $Y$, but it is not a peak point. Therefore we have constructed the required example.

\section{References}

[1] G. R. Allan, A spectral theory for locally convex algebras, Proc. London Math. Soc. (3) 15 (1965) pp. 399-421.

[2] R. Arens and A. P. Calderón, Analytic functions of several Banach algebra elements, Ann. of Math. 62 (1955) pp. 204-216.

[3] N. Bourbaki, Espaces vectoriels topologiques, Chapitres 1 et 2, Paris 1953.

[4] - Théories spectrales, Chapitres 1 et 2, Paris 1967.

[5] T. W. Gamelin, Uniform algebras, N. J. 1969.

[6] B. Gramsch, Funktionallialkül mehrerer Veränderlichen in lokalbeschränkten Algebren, Math. Ann. 174 (1967) 311-342.

[7] H. Komatsu, Projective and injective limits of weakly compact sequences of locally convex spaces, J. Math. Soc. Japan 19 (1967) pp. 366-383.

[8] E. A. Michael, Locally multiplicatively convex topological algebras, Mem. Amer. Math. Soc. 11 (1952).

[9] C. E. Rickart, The maximal ideal space of functions locally approximable in a function algebra, Proc. Amer. Math. Soc. 17 (1966) pp. 1320-1326.

[10] A. P. Robertson and W. J. Robertson, Topological vector spaces, Cambridge, 1966.

[11] L. Waelbroeck, Le calcul symbolique dans les algèbres commutatives, J. Math. Pures Appl. 33 (1954) pp. 147-186.

[12] - About a spectral theorem, Function algebras (ed. F. T. Birtel), Scott Foresman, Glenview, III, 1966.

[13] W. 亡̇elazko, Metric generalizations of Banach algebras, Rozprawy Matematyczne no. 47, Warsaw, 1965.

UNIVERSITY OF CAMBRIDGE

Reçu par la Rédaction le 12.7.1970 